\title{
Topological Structure of Riesz Sequence Spaces
}

\author{
Merve Temizer Ersoy ${ }^{1, *}$, Bilal Altay ${ }^{2}$, Hasan Furkan ${ }^{1}$ \\ ${ }^{1}$ Department of Mathematics, Kahramanmaras Sutcu Imam University, Kahramanmaras, Turkey \\ ${ }^{2}$ Department of Primary Education, Inonu University, Malatya, Turkey
}

Email address:

mervetemizer@hotmail.com (M. T. Ersoy), bilal.altay@inonu.edu.tr (B. Altay), hasanfurkan@hotmail.com (H. Furkan)

${ }^{*}$ Corresponding author

\section{To cite this article:}

Merve Temizer Ersoy, Bilal Altay, Hasan Furkan. Topological Structure of Riesz Sequence Spaces. Applied and Computational Mathematics. Vol. 7, No. 1, 2018, pp. 26-30. doi: 10.11648/j.acm.20180701.14

Received: December 29, 2017; Accepted: January 12, 2018; Published: January 20, 2018

\begin{abstract}
In this paper, to be the Riesz matrix is symbolized by $R$, it is defined the spaces $l_{\infty}(R), c(R)$ and $c_{0}(R)$, where for instance $c_{0}(R)=\left\{x=\left(x_{k}\right) \in w \mid \lim _{n} \frac{1}{P_{n}} \sum_{k=1}^{n} p_{k} x_{k}=0\right\}$ and computed its duals ( $\alpha$-dual, $\beta$-dual and $\gamma$-dual). Furthermore, it is investigated topological structure of $c_{0}(R)$ and determined necessary and sufficient conditions for a matrix $C$ to map $c_{0}(R)$, or $c_{0}$ into $c_{0}$ or $c_{0}(R)$.
\end{abstract}

Keywords: Topological Sequence Space, Banach Spaces, $\alpha$-Dual, $\beta$-Dual

\section{Introduction}

The study of sequence spaces is in fact a special case of the more general study of function spaces if the domain is restricted to the set of natural numbers $\mathrm{N}$. The set $w$ of all functions from the natural numbers $\mathrm{N}$ to the field $\mathrm{K}$ of real $\mathrm{R}$ or complex numbers $\mathrm{C}$, can be turned into a vector space. In other words, let $w$ be the set of all (real-or) complex valued sequences $\left\{x_{n}\right\}_{n \in N}$, i.e., $x_{n} \in C$ under the operations of pointwise addition and scalar multiplication given by

$$
\left\{x_{n}\right\}_{n \in N}+\left\{y_{n}\right\}_{n \in N}=\left\{x_{n}+y_{n}\right\}_{n \in N}
$$

and $\lambda\left\{x_{n}\right\}_{n \in N}=\left\{\lambda x_{n}\right\}_{n \in N}$, for every $x_{n}, y_{n} \in C$ and scalar $\lambda$ form a vector space over C. Any subspace $x$ of $w$ is then called a sequence space. In other words, a sequence space is a vector space whose elements are infinite scalar sequences of real or complex numbers and is closed under the coordinate wise addition and scalar multiplication. If it is closed under coordinate wise multiplication as well, it is called a sequence algebra. Sequence spaces when equipped with a linear topology form topological vector spaces, [1].

The spaces $l_{\infty}, c$ and $c_{0}$ are the linear spaces of complex bounded, convergent and null sequences respectively, i.e., $1_{\infty}=$ $\left\{\mathrm{x}=\left(\mathrm{x}_{\mathrm{k}}\right) \in \mathrm{w}: \sup _{\mathrm{k}}\left|\mathrm{x}_{\mathrm{k}}\right|<\infty\right\}, \mathrm{c}=\left\{\mathrm{x}=\left(\mathrm{x}_{\mathrm{k}}\right) \in \mathrm{w}: \lim _{\mathrm{k}} \mathrm{x}_{\mathrm{k}}\right.$ exists $\}$, $\mathrm{c}_{0}=\left\{\mathrm{x}=\left(\mathrm{x}_{\mathrm{k}}\right) \in \mathrm{w}: \lim _{\mathrm{k}} \mathrm{x}_{\mathrm{k}}=0\right\}$.

The multipliers from $X$ into $Y$ are given by

$$
X^{Y}=\{y \in w \mid x y \in Y, \forall x \in X\}
$$

for $X, Y \subset w$, where $x y$ is the coordinate wise product, i.e., $x y=\left\{x_{k} y_{k}\right\}_{k \in N}$. We notates

$$
\left(X^{Y}\right)^{Z}=X^{Y Z}=\left\{y \in w \mid x y \in Z, \forall x \in X^{Y}\right\}
$$

for $\mathrm{X}, \mathrm{Y}, \mathrm{Z} \subset \mathrm{w}$. A sequence space $\mathrm{X}$ is called $\mathrm{Y}$-space if $\mathrm{X}=\mathrm{X}^{\mathrm{Y}}$ ${ }^{\mathrm{Y}}$. Classical $\alpha$-, $\beta$ - and $\gamma$-duals of $\mathrm{X}$ are given by $\mathrm{X}^{1,} \mathrm{X}^{\mathrm{cs}}$ and $\mathrm{X}^{\mathrm{bs}}$, respectively, where

$$
\begin{gathered}
l=\left\{x=\left(x_{k}\right) \in w\left|\sum_{k}\right| x_{k} \mid<\infty\right\}, \\
c s=\left\{x=\left(x_{k}\right) \in w \mid \sum_{k} x_{k} \text { is convergent }\right\}, \\
b s=\left\{x=\left(x_{k}\right) \in w\left|\sup _{n}\right| \sum_{k} x_{k} \mid<\infty\right\} .
\end{gathered}
$$

These are Banach spaces with their natural norms. We know that $\varphi \subset X^{\alpha} \subset X^{\beta} \subset X^{\gamma}$. If $X \subset Y$ then $Y^{\zeta} \subset X^{\zeta}$ and for every $X$ we have $X^{\zeta}=X^{\zeta \zeta \zeta}, X \subset X^{\zeta \zeta}$, where $\zeta$ is one of the $\alpha$-, $\beta$ - or $\gamma$-duals. [2-4].

Let $\mathrm{X}$ and $\mathrm{Y}$ be two sequence spaces and let $A=\left(a_{n k}\right)$ be an infinite matrix of complex numbers $a_{n k}$ where $n, k \in \mathrm{N}$. Then we say that A defines a matrix transformation from $X$ 
into $Y$ and we denote it by writing $A: X \rightarrow Y$ if for every sequence $x=\left(x_{k}\right) \in X$, the sequence $A x=\{(A x)\}$, the $A$-transform of $x$, is in $Y$, where

$$
(A x)_{n}=\sum_{k} a_{n k} x_{k},\left(n \in N, x \in D_{00}(A)\right)
$$

and by $D_{00}(A)$ denotes the subspace of $w$ consisting of $x \in w$ for which the sum exists as a finite sum. By $(X: Y)$, we denote the class of all matrices $A$ such that $A: X \rightarrow Y$. Thus $A \in(X: Y)$ if and only if the series on the right side of (1) convergent for each $n \in \mathrm{N}$ and each $x \in X$ and we have $A x=\left\{(A x)_{n}\right\}_{n \in \mathrm{N}} \in Y$ for all $x \in X$. For an arbitrary sequence space $X$, the matrix domain $X_{A}($ or $X(A))$ of an infinite matrix $\mathrm{A}$ in $X$ is defined by $X_{A}=\{x$ $\in w: A x \in X\}$, which is a sequence space.

Kizmaz [5] defined the sequence spaces

$$
\begin{aligned}
l_{\infty}(\Delta) & =\left\{x=\left(x_{k}\right) \mid \Delta x \in l_{\infty}\right\}, \\
c(\Delta) & =\left\{x=\left(x_{k}\right) \mid \Delta x \in c\right\}, \\
c_{0}(\Delta) & =\left\{x=\left(x_{k}\right) \mid \Delta x \in c_{0}\right\},
\end{aligned}
$$

where $\Delta x=\left(\Delta x_{k}\right)=\left(x_{k}-x_{k+1}\right)$, and showed that these are Banach spaces with norm $\|x\|=\left|x_{1}\right|+\|\Delta x\|_{\infty}$. After then Et [6] defined the sequence spaces $l_{\infty}\left(\Delta^{2}\right), c\left(\Delta^{2}\right), c_{0}\left(\Delta^{2}\right)$ where $\Delta^{2} x=\left(\Delta^{2} x_{k}\right)=\left(\Delta x_{k}-\Delta x_{k+1}\right)$, and showed that these are Banach spaces with norm $\|x\|_{1}=\left|x_{1}\right|+\left|x_{2}\right|+\left\|\Delta^{2} x\right\|_{\infty}$. Recently Pooja [7] study on $\mathrm{m}^{\text {th }}$ difference sequence spaces and Khan [8] works on some inclusion relations between the difference sequence spaces defined by sequence of moduli.. In addition, similar topics are studied and genaralized by some author [9-10].

\section{Main Results}

Let $p=\left(p_{k}\right)$ and $\left(P_{n}\right)$ be given $\mathrm{p}_{0}>0, p_{k} \geq 0(\forall \mathrm{k}, \mathrm{n} \in$ $\mathrm{N}), \quad P_{n}=\sum_{k=0}^{n} p_{k}$. Let the matrix $R=R_{p}=(R, p)=$ $\left(R, p_{n}\right)=r_{n k}$ defined by

$$
\left(r_{n k}\right)=\left\{\begin{array}{c}
\frac{p_{k}}{P n}, k \leq n \\
0, \text { otherwise }
\end{array}\right.
$$

is called a Riesz matrix (associated with the sequence $\mathrm{p}$ ) and

$$
\left(r^{-1}{ }_{n k}\right)=\left\{\begin{array}{c}
(-1)^{n-k} \frac{P_{k}}{p_{n}}, k \leq n \leq k+1 \\
0, k+1<n
\end{array}\right.
$$

is called inverse matrix of Riesz matrix. Also, for $R x=y$, we get

$$
y_{n}=\frac{1}{P_{n}} \sum_{k=1}^{n} p_{k} x_{k}
$$

and

$x_{n}=\frac{1}{p_{n}}\left(P_{n} y_{n}-P_{n-1} y_{n-1}\right)$ or $x_{n}=\sum_{k=n-1}^{n}(-1)^{n-k} \frac{P_{k}}{p_{n}} y_{k}$.

Now we define

$$
\begin{gathered}
l_{\infty}(R)=\left\{x=\left(x_{k}\right): \sup _{n}\left|\frac{1}{P_{n}} \sum_{k=1}^{n} p_{k} x_{k}\right|<\infty\right\}, \\
c(R)=\left\{x=\left(x_{k}\right): \lim _{n}\left|\frac{1}{P_{n}} \sum_{k=1}^{n} p_{k} x_{k}-l\right|=0, l \in R\right\}, \\
c_{0}(R)=\left\{\begin{array}{c}
\left.x=\left(x_{k}\right): \lim \left(\frac{1}{P_{n}} \sum_{k=1}^{n} p_{k} x_{k}\right)=0\right\} .
\end{array}\right.
\end{gathered}
$$

These spaces are sequence spaces and are normed spaces with norm $\|x\|_{E(R)}=\sup \left|R_{n} x\right|$ where $E$ is one of the sequence spaces $l_{\infty}, c, c_{0}$ respectively.

Teorem 2.1. Let $\mathrm{X}$ be a normed space and $x=\left(x_{k}\right)$ be a sequence in $\mathrm{X}$. We also define the operator

$$
\begin{gathered}
T: c_{0}(R) \rightarrow c_{o} \\
x \rightarrow T x=R x=\frac{1}{P_{n}} \sum_{k=1}^{n} p_{k} x_{k}
\end{gathered}
$$

Then $T$ is a linear isometry.

Proof. Let $\forall x, \quad x^{\prime} \in c_{0}(R)$ and $\alpha \in \mathrm{K}$. The equality

$$
\mathrm{T}\left(\alpha \mathrm{x}+x^{\prime}\right)=\mathrm{R}\left(\alpha \mathrm{x}+x^{\prime}\right)=\alpha \mathrm{Rx}+\mathrm{R} x^{\prime}=\alpha \mathrm{T} \mathrm{x}+\mathrm{T} x^{\prime}
$$

holds. From this, we get that the operator $T$ is a linear. If the equality $T x=T x^{\prime}$ holds, then we have

$$
\frac{1}{P_{n}} \sum_{k=1}^{n} p_{k}\left(x_{k}-x_{k}^{\prime}\right)=0 .
$$

By induction method, we have $x_{n}=x^{\prime}{ }_{n}$ for all $n \in \mathrm{N}$. Therefore the operator $T$ is injective. Furthermore, because the operator $T$ is bijective, we get immediately that $c_{0}(R) \cong c_{0}$.

Theorem 2.2. $\left(E(R),\|.\|_{E(R)}\right)$ are normed spaces where $E$ is one of the sequence space $l_{\infty}, c, c_{0}$.

Proof. Let $E$ be $c_{0}$ sequence space and $\alpha \in \mathrm{K}$.

(i) $\|x\|_{c_{0}(R)}=0 \Leftrightarrow \sup _{n}\left|\frac{1}{P_{n}} \sum_{k=1}^{n} p_{k} x_{k}\right|=0$

$$
\begin{aligned}
& \Leftrightarrow \sup \frac{1}{P_{n}} \sum_{k=1}^{n} p_{k} x_{k}=0 \\
& \Leftrightarrow x=0 \text { for all } n \in N .
\end{aligned}
$$

(ii) $\|a x\|_{c_{0}(R)}=\|a R x\|_{c_{0}}=\sup \left|\frac{1}{P_{n}} \sum_{k=1}^{n} a p_{k} x_{k}\right|$

$$
=|a| .\|R x\|_{c_{0}}=|a| . \|\left. x\right|_{c_{0}(R)}
$$

(iii) For $x, x^{\prime} \in c_{0}(R)$,

$$
\begin{aligned}
\| x & +x^{\prime}\left\|_{c_{0}(R)}=\right\| R\left(x+x^{\prime}\right) \|_{c_{0}} \\
& =\sup _{n}\left|\frac{1}{P_{n}} \sum_{k=1}^{n} p_{k}\left(x_{k}+x^{\prime}{ }_{k}\right)\right|
\end{aligned}
$$




$$
\begin{aligned}
\leq \sup _{n} & \left|\frac{1}{P_{n}} \sum_{k=1}^{n} p_{k} x_{k}\right|+\sup _{n}\left|\frac{1}{P_{n}} \sum_{k=1}^{n} p_{k} x^{\prime}{ }_{k}\right| \\
& \leq\|R x\|_{c_{0}}+\left\|R x^{\prime}\right\|_{c_{0}} \\
& \leq\|x\|_{c_{0}(R)}+\left\|x^{\prime}\right\|_{c_{0}(R)} .
\end{aligned}
$$

From (i), (ii) and (iii) we immediately get $\left(c_{0}(R),\|\cdot\|_{c_{0}(R)}\right)$ is a normed spaces. Similarly we can prove for other sequence spaces.

Teorem 2.3. The normed spaces $\left(c_{0}(R),\|\cdot\|_{c_{0}(R)}\right)$ is a Banach space.

Proof. Let $\left(x^{n}\right)$ be a Cauchy sequence in $c_{0}(R)$, where $x^{n}=$ $\left(x^{n}\right)=\left(x_{1}^{n}, x_{2}^{n}, \cdots\right) \in c_{0}(R)$, for each $n \in \mathrm{N}$. Then for each $\varepsilon>$ 0 , there exists $N=N(\varepsilon)$, such that for all $n, m \geq N$,

$$
\begin{gathered}
\left\|x^{n}-x^{m}\right\|_{c_{0}(R)}=\left\|R\left(x^{n}-x^{m}\right)\right\|_{c_{0}} \\
=\left\|R x^{n}-R x^{m}\right\|_{c_{0}}<\varepsilon .
\end{gathered}
$$

Therefore we obtain that $R x^{n}$ is a Cauchy sequence in $c_{0}$ whence by $\left(c_{0},\|\cdot\|_{c_{0}}\right)$ the Banach space, it converges to $(R x)$ say, i.e., there exists $\lim R x^{n}=R x \in c_{0}$. Now we must show that $x \in c_{0}(R)$. We have

$$
\lim R x^{n}=R x \in c_{0} \Leftrightarrow\left\|R x^{n}-R x\right\|_{c_{0}} \rightarrow 0 .
$$

Thus, we get

$$
\left\|R x^{n}-R x\right\|_{c_{0}}=\left\|R\left(x^{n}-x\right)\right\|_{c_{0}}=\left\|\left(x^{n}-x\right)\right\|_{c_{0}(R)} \rightarrow 0 .
$$

So, it is corrected that $x^{n} \rightarrow x \in c_{0}(R)$. This implies $\left(c_{0}(R),\|\cdot\|_{c_{0}(R)}\right)$ is a Banach space.

We shall quote some lemmas which are needed in proving our theorems concerning the $\alpha, \beta, \gamma$ duals of the Riesz sequence spaces.

Lemma 2.1. Let $R$ be a Riesz matrix. Then $R \in\left(c_{0}, c_{0}\right)$ if and only if

(i) $\lim _{n} r_{n k}=0$,

(ii) $\sup \sum_{k}\left|r_{n k}\right|<\infty$.

Lemma 2.2. Let $\mathrm{R}$ be a Riesz matrix. Then $\mathrm{R} \in\left(\mathrm{c}, \mathrm{c}_{0}\right)$ if and only if

(i) $\lim _{n} r_{n k}=0$,

(ii) $\sup \sum_{k}\left|r_{n k}\right|<\infty$,

(iii) $\lim \sum_{k} r_{n k}=0$.

Lemma 2.3. Let $R$ be a Riesz matrix. Then $R \in\left(c_{0}, c\right)$ if and only if

(i) $\lim _{n} r_{n k}$ exists,

(ii) $\sup \sum_{k}\left|r_{n k}\right|<\infty$.

Lemma 2.4. Let $R$ be a Riesz matrix. Then $R \in\left(c_{0}, l_{\infty}\right)$ if and only if

(i) $\sup \sum_{k}\left|r_{n k}\right|<\infty$.

Lemma 2.5. Let $R$ be a Riesz matrix. Then $R \in\left(c_{0}, l\right)$ if and only if

(ii) $\sup \sum_{n=1}^{\infty}\left|\sum_{k} r_{n k}\right|<\infty$.

Teorem 2.4. Let $R$ be a Riesz matrix and $P_{n} \rightarrow \infty(n \rightarrow 0)$ where $P_{n}=\sum_{k=0}^{n} p_{k}$ for each $n \in \mathrm{N}$, then the inclusion $c_{0} \subset$ $c_{0}(R)$ holds.
Proof. Let $R$ be a Riesz matrix and $x=\left(x_{k}\right)$ be a sequence in $c_{0}$. Then the following statement is obvious.

$$
x \in c_{0} \Rightarrow R x \in c_{0} \text { and } R x \in c_{0} \Leftrightarrow R \in\left(c_{0}, c_{0}\right) .
$$

By Lemma 2.1, we have $R \in\left(c_{0}, c_{0}\right) \Leftrightarrow$

(i) $\lim _{n} r_{n k}=0$,

(ii) $\sup \sum_{k}\left|r_{n k}\right|<\infty$.

Now we show the correctness of these statements.

$$
\lim _{\mathrm{n}} r_{n k}=\lim \frac{p_{k}}{P_{n}}=p_{k} \lim \frac{1}{P_{n}}=p_{k} \cdot 0=0,
$$

and

$$
\sup \sum_{k}\left|r_{n k}\right|=\sup \sum\left|\frac{p_{k}}{P_{n}}\right|=1<\infty .
$$

This implies $R \in\left(c_{0}, c_{0}\right)$ such that for each $x \in c_{0}(R)$. This completes the proof.

Theorem 2.5. Let $R$ be a Riesz matrix and $\sup \left|P_{n}\right|<\infty$, then the inclusion $c_{0}(R) \subset c_{0}$ holds.

Proof. Let $R^{-1}$ be inverse of Riesz matrix and $x=\left(x_{k}\right)$ be a sequence in $c_{0}(R)$. We need to find that $x \in c_{0}$ or equivalent we need to find that $R^{-1} \in\left(c_{0}, c_{0}\right)$. By Lemma 2.1, we can easily show the following statements.

$$
\begin{gathered}
\lim _{n} r^{-1}{ }_{n k}=\lim (-1)^{n-k} \frac{P_{k}}{p_{n}}=0, \\
\sup \sum_{k}\left|r_{n k}^{-1}\right|=\sup \sum_{k}\left|(-1)^{n-k} \frac{P_{k}}{p_{n}}\right|=1<\infty .
\end{gathered}
$$

This implies $R^{-1} \in\left(c_{0}, c_{0}\right)$ such that for each $x \in c_{0}$. Hence the proof is completed.

Theorem 2.6. Let $R$ be a Riesz matrix. Then we have

(i) $\mathrm{c}_{0} \subset 1_{\infty}(\mathrm{R})$ if and only if $\sup \sum_{k}\left|r_{n k}\right|<\infty$,

(ii) The inclusion $\mathrm{c} \subset \mathrm{c}_{0}(\mathrm{R})$ is invalid.

Proof. (i) If the inclusion $c_{0} \subset l_{\infty}(R)$ holds, then the necessary and sufficient condition is that $\mathrm{R} \in\left(c_{0}, l_{\infty}\right)$. By Lemma 2.4, we must show that

$$
\sup \sum_{k}\left|r_{n k}\right|<\infty .
$$

It is seen by Theorem 2.4. Hence, we say the inclusion $c_{0} \subset$ $l_{\infty}(R)$ holds.

(ii) If the inclusion $\mathrm{c} \subset \mathrm{c}_{0}(\mathrm{R})$ holds, then the necessary and sufficient condition is that $R \in\left(c, c_{0}\right)$. By Lemma2.2, one can show that the validity of the options (i) and (ii). However, the part of (iii) is not provided, i.e., $\lim \sum_{k} r_{n k}=1 \neq 0$.Thus, we obtain that the inclusion $c \subset c_{0}(\mathrm{R})$ is invalid.

Theorem 2.7. Let $R$ be a Riesz matrix and $x=\left(x_{k}\right)$ be a sequence in $c_{0}(R), y=\left(y_{k}\right)$ be a sequence in $c_{0}$. Then we have 
(i)

$$
\left(c_{0}(R)\right)^{\alpha}=\left\{a=\left(a_{k}\right) \in w: \sup \sum_{n=1}^{\infty}\left|\sum_{k}(-1)^{n-k} a_{n} \frac{P_{k}}{p_{n}}\right|<\infty\right\},
$$

(ii)

$$
\left(c_{0}(R)\right)^{\beta}=\left(c_{0}(R)\right)^{\gamma}=\left\{a=\left(a_{k}\right) \in w:\left(\Delta\left(\frac{a_{k}}{p_{k}}\right) p_{k}\right) \in l,\left(a_{n} \frac{P_{n}}{p_{n}}\right) \in l_{\infty}\right\}
$$

Proof. (i) Let us take any $a=\left(a_{k}\right) \in w$. We easily derive with

$$
a x=\left(a_{k} x_{k}\right)=\left(a_{k} \sum_{i=1}^{k}(-1)^{k-i} \frac{P_{i}}{p_{k}} y_{i}\right)=\sum_{i=1}^{k}(-1)^{k-i} a_{k} \frac{P_{i}}{p_{k}} y_{i}=(T y)_{k}
$$

For all $k \in N$, where $T=\left(t_{n k}\right)$ is defined by

$$
t_{n k}=\left\{\begin{array}{c}
(-1)^{n-k} a_{n} \frac{P_{k}}{p_{n}}, n-1 \leq k \leq n \\
0, \text { otherwise }
\end{array}\right.
$$$$
\Leftrightarrow\left\{a=\left(a_{k}\right) \in w: \sup \sum_{n=1}^{\infty}\left|\sum_{k}(-1)^{n-k} a_{n} \frac{P_{k}}{p_{n}}\right|<\infty\right\} .
$$

(ii) Let $x \in c_{0}(R)$ and $a \in w$. Then $y=R x \in c_{0}$, We get

We also define the linear operator

$$
\begin{gathered}
T: c_{0} \rightarrow l \\
y \rightarrow T y=a x \in l .
\end{gathered}
$$

By Lemma 2.5, we get

(i) $a \in\left(c_{0}(R)\right)^{\alpha} \Leftrightarrow a x \in l, \forall x \in\left(c_{0}\right) R$,

$$
\begin{gathered}
\Leftrightarrow \forall R x=y \in c_{0}, T y \in l \\
\Leftrightarrow T \in\left(c_{0}, l\right) \Leftrightarrow \sup \sum_{n=1}^{\infty}\left|\sum_{k} t_{n k}\right|<\infty \quad \begin{array}{r}
a_{n} \frac{p_{n}}{p_{n}}, k=n \\
0, \text { otherwise }
\end{array} \\
a \in\left(c_{0}(R)\right)^{\beta} \Leftrightarrow a x \in c s, \forall x \in c_{0}(R) \Leftrightarrow \sum_{k=1}^{n} a_{k} x_{k} \in c, \forall x \in c_{0}(R) \\
\Leftrightarrow(C y)_{n} \in c, \forall y \in c_{0} \Leftrightarrow C \in\left(c_{0}, c\right) .
\end{gathered}
$$

By Lemma 2.3, we get

$$
\left(c_{0}(R)\right)^{\beta}=\left\{a=\left(a_{k}\right) \in w:\left(\Delta\left(\frac{a_{k}}{p_{k}}\right) p_{k}\right) \in l \text { and }\left(a_{n} \frac{P_{n}}{p_{n}}\right) \in l_{\infty}\right\}
$$

On the other hand, $\left(c_{0}(R)\right)^{\gamma}$ can be found in a similar way.

Theorem 2.8. Let $(X, Y)$ denote the set of all infinite matrices $C$ which map X into $\mathrm{Y}$. Then we have $C \in\left(c_{0}(R), c_{0}\right)$ if and only if

(i) $\mathrm{D}^{(\mathrm{m})} \in\left(\mathrm{c}_{0}, \mathrm{c}\right)$,

(ii) $\mathrm{D} \in\left(\mathrm{c}_{0}, \mathrm{c}_{0}\right)$

where the matrix $D$ depends on the matrix $C,(\forall m \in \mathrm{N})$.

Proof. If $x \in c_{0}(R)$ then $y=R x \in c_{0} \Rightarrow x=R^{-1} y$, we have

$$
\begin{gathered}
C_{n}^{(m)} x=\sum_{k=1}^{m} c_{n k} x_{k} \\
=\sum_{k=1}^{m} c_{n k}\left(\frac{1}{p_{k}}\left(P_{k} y_{k}-P_{k-1} y_{k-1}\right)\right)
\end{gathered}
$$

$$
=\sum_{k=1}^{m-1}\left(\frac{c_{n k}}{p_{k}}-\frac{c_{n k+1}}{p_{k}}\right) P_{k} y_{k}+\frac{c_{n m}}{p_{m}} P_{m} y_{m}=D_{n}^{(m)} y
$$

Now we also define the matrix:

$$
d_{n k}^{(m)}=\left\{\begin{array}{c}
\left(\frac{c_{n k}}{p_{k}}-\frac{c_{n k+1}}{p_{k}}\right) P_{k}, k<m \\
\frac{c_{n m}}{p_{m}} P_{m}, k=m \\
0, \text { otherwise }
\end{array}\right.
$$

Hence, by Lemma 2.1 and Lemma 2.3, we get

(i) $\mathrm{D}^{(\mathrm{m})} \in\left(\mathrm{c}_{0}, \mathrm{c}\right) \Leftrightarrow$

(1) $\sup \sum_{k}\left|d_{n k}\right|=\sup \sum_{k}\left|\frac{c_{n k}}{p_{k}}-\frac{c_{n k+1}}{p_{k}}\right| P_{k}<\infty \quad$ and 
$\left|\frac{c_{n m}}{p_{m}} P_{m}\right|<\infty$

(2) $\lim _{n} d_{n k}=\lim _{n}\left|\frac{c_{n k}}{p_{k}}-\frac{c_{n k+1}}{p_{k+1}}\right| P_{k}$ exists.

(ii) $D \in\left(c_{0}, c_{0}\right) \Leftrightarrow$

(1) $\sup \sum_{k}\left|d_{n k}\right|=\sup \sum_{k}\left|\frac{c_{n k}}{p_{k}}-\frac{c_{n k+1}}{p_{k}}\right| P_{k}<\infty \quad$ and $\left|\frac{c_{n m}}{p_{m}} P_{m}\right|<\infty$

(2) $\lim _{n} d_{n k}=\lim _{n}\left|\frac{c_{n k}}{p_{k}}-\frac{c_{n k+1}}{p_{k+1}}\right| P_{k}=0$.

Hence, it is clear that $\mathrm{C} \in\left(\mathrm{c}_{0}(\mathrm{R}), \mathrm{c}_{0}\right)$. Conversely, this is obtained by the same kind of argument.

Theorem 2.9. Let $\mathrm{C}$ be a matrix. Then we have $C \in$ $\left(c_{0}, c_{0}(R)\right)$ if and only if

(i) $\sup \sum_{k=1}^{\infty}\left|\frac{1}{P_{n}} \sum_{i=1}^{n} p_{k} c_{i k}\right|<\infty$,

(ii) $\lim \frac{1}{P_{n}} \sum_{i=1}^{n} p_{i} c_{i k}=0$.

Proof. Let $C \in\left(c_{0}, c_{0}(R)\right)$. For each $x \in c_{0}, C x$ exists and we have $C x \in c_{0}(R) \Rightarrow R C x \in c_{0} \Rightarrow R C \in\left(c_{0}, c_{0}\right)$.

If $\mathrm{D}$ is a matrix RC, then we can take $d_{n k}=\frac{1}{P_{n}} \sum_{i=1}^{n} p_{i} c_{i k}$.

By Lemma 2.1, there is a matrix $\mathrm{C}$ that provides the following statements such that

$\sup \sum_{k=1}^{\infty}\left|\frac{1}{P_{n}} \sum_{i=1}^{n} p_{k} c_{i k}\right|<\infty$ and $\lim \frac{1}{P_{n}} \sum_{i=1}^{n} p_{i} c_{i k}=0$.

This is the desired result. In addition, the sufficient condition is trivial.

\section{Conclusion}

In this paper, to be the Riesz matrix is symbolized by $R$, we define the spaces $l_{\infty}(R), c(R)$ and $c_{0}(R)$ and compute duals ( $\alpha$-dual, $\beta$-dual and $\gamma$-dual) of $c_{0}(R)$. Also, it is shown that if the matrix $\mathrm{R}$ are a regular matrix and $\sup \left|P_{n}\right|<\infty$ then $c_{0}=c_{0}(R)$. Finally, we investigate topological structure of $c_{0}(R)$ and determine necessary and sufficient conditions for a matrix $C$ to map $c_{0}(R)$, or $c_{0}$ into $c_{0}$ or $c_{0}(R)$.

\section{Acknowledgements}

M. Temizer Ersoy has been supported by the Scientific and Technological Research Council of Turkey (TUBITAK Programme, 2228-B).

\section{References}

[1] N. P. Pahari, "Some classical sequence spaces and their topological structures, Jacem. (2015), vol 1.

[2] J. Boos, "Classical and Modern Methods in Summability", Oxford University Press. New York, Oxford, (2000).

[3] A. Wilansky, "Summability Through Functional Analysis", North Holland, (1984).

[4] A. Wilansky, "Functional Analysis", Blaisdell Press, (1964).

[5] H. Kizmaz, "On Certain Sequence Spaces", Canad. Math. Bull. Vol 24 (2) (1981), pp. 169- 176.

[6] M. Et, “On Some Difference Sequence Spaces”, Tr. J. of Math. 17 (1993), pp. 18-24.

[7] V. Pooja, "M th Difference Sequence Spaces", IJCMS. vol 5 (2016).

[8] V. A. Khan, "Some inclusion relations between the difference sequence spaces defined by sequence of moduli", J. Indian Math Soc. Vol 73 (2016), pp. 77-81.

[9] F.Basar, M. Kirisci, "Almost convergence and generalized difference matrix", Comput. Math. Appl. Vol 61 (3) (2011), pp. 602-611.

[10] B. Altay, "On the space of p-summable difference sequences of order m", Stud. Sci. Math. Hungar. Vol 43 (4) (2006), pp. 387-402. 